\title{
On the contribution of direct intermacromolecular interactions to the viscosity of a suspension of hard spheres
}

\author{
George D. J. Phillies \\ Department of Chemistry, University of Michigan, Ann Arbor, Michigan 48109 \\ (Received 10 October 1978) \\ The contribution of the contact interaction to the shear and bulk viscosities $\eta$ and $\eta_{\nu}$ of a suspension of \\ hard spheres is evaluated by use of the Green-Kubo integral forms. To lowest (second) order in the \\ volume fraction $\phi, \eta=\eta_{0}\left(1+13.44 \phi^{2}\right)$ and $\eta_{v}=\eta_{v 0}\left(1+4.32 \eta_{0} \phi^{2}\right)$. Since [J. Chem. Phys. 67, 4690 \\ (1977)] direct interactions contribute to the drag coefficient $f$ in first order in the density, a sphere \\ suspension whose dissipative properties include a substantial contribution from direct intermacromolecular \\ interactions is predicted not to show Debye-type behavior, i.e., for such a system $f / \eta$ will not be \\ independent of concentration.
} tion

$$
\delta f \hat{\mathrm{v}}=\frac{-\beta}{v_{0}}\left\langle\int_{0}^{\infty} d t \mathrm{~F}(0) \mathrm{F}(t) \cdot \mathbf{v}(t) d t\right\rangle
$$

was used to calculate the contribution $\delta f$ of direct intermacromolecular interactions to the Stokes-law drag coefficient $f$ of a single probe macromolecule. Here $\mathbf{F}(t)$ is the fluctuating force on the probe due to direct interactions between the macromolecules, $\nabla(t)=v_{0} \hat{v}$ is the velocity of the probe, $\beta=\left(K_{B} T\right)^{-1}$, and the brackets indicate an ensemble average. $F(t)$ is determined by concentration fluctuations around the probe. For a suspension of hard spheres, the first order contribution of direct interactions to $f$ was found to be

$$
f=f_{0}\left(1+\frac{8}{3} \varnothing\right)
$$

$f_{0}$ and $\varnothing$ being the zero-concentration drag coefficient and the volume fraction of the spheres, respectively.

In this note, the same approximations are used to evaluate the contribution of the hard sphere interaction to the shear and bulk viscosities $\eta$ and $\eta_{v}$ of a hard sphere suspension. The viscosities are obtained from the Green-Kubo forms ${ }^{2}$

$$
\begin{aligned}
& \eta=\frac{\beta}{V} \int_{0}^{\infty} d t\left\langle\sigma_{x y}(0) \sigma_{x y}(t)\right\rangle, \\
& \eta_{v}=\frac{\beta}{V} \int_{0}^{\infty} d t\left\langle\sigma_{x x}(0) \sigma_{y y}(t)\right\rangle,
\end{aligned}
$$

where

$$
\sigma_{a b}(t)=\sum_{i=1}^{N}\left\{p_{i a}(t) p_{i b}(t) m_{i}^{-1}+r_{i a}(t) F_{T i b}(t)\right\}-\left\langle\sigma_{a b}\right\rangle
$$

The subscripts $a, b$ refer to the cartesian axes $x, y, z$; $p_{i a}(t), r_{i a}(t)$, and $F_{r i a}(t)$ are the $a$ th component of the momentum, position, and total applied force for particle $i$ at time $t$. The force $\mathrm{F}_{T_{1}}(t)$ is composed of a part $\mathrm{F}_{8}(t)$ due to the solvent and a part $F_{1}(t)$ due to direct intermacromolecular interactions, the latter being ${ }^{1}$

$$
\mathrm{F}_{i}(t)=\frac{i K_{B} T}{(2 \pi)^{3}} \sum_{k} \mathrm{k} a_{\mathbf{z}}(t) h^{(2)}(\mathrm{k}) e^{i \mathbf{k} \cdot \mathrm{r}_{i}(t)},
$$

where $a_{\mathbf{k}}(t)$ is the kth spatial fourier component of the concentration at time $t, h^{(2)}(\mathrm{k})=\int d \mathbf{r}\left(g^{(2)}(\mathbf{r})-1\right) \exp (i \mathrm{k} \cdot \mathbf{r})$, and $g^{(2)}(r)$ is the two-body equilibrium radial distribution function. For diffusing macromolecules, whether interacting or not, ${ }^{3}$

$$
\left\langle a_{\mathbf{k}}(0) a_{-\mathbf{k}}(t)\right\rangle=\left\langle\left|a_{\mathbf{k}}(0)\right|^{2}\right\rangle e^{-D_{m^{k^{2} t}},},
$$

where $D_{m}$ is the mutual diffusion coefficient.

It is convenient to assume that the direct and solventbased forces $F_{t}(t)$ and $F_{s i}(t)$ are uncorrelated. In this case, cross-terms between $F_{s i}(t)$ and $F_{i}$ vanish; the contribution of direct interactions to the viscosities then arises from terms of $\sigma_{a b}$ which depend solely on $F_{q}$ 。 Since $F_{s t}$ and $F_{i}$ have greatly different correlation times, this assumption is not unreasonable; however, it clearly cannot be exact. The momentum $p_{i}$ is determined by the forces acting on particle $i$ at all previous times; for time intervals comparable with the (long) correlation time of $\mathrm{F}_{i},\left\langle\mathrm{p}_{i}(t) \mathrm{F}_{i}(t+\tau)\right\rangle$ and $\left\langle\mathrm{p}_{i}(t) \mathrm{p}_{i}(t+\tau)\right\rangle$ are therefore nonvanishing. For simplicity, possible corrections due to hydrodynamic interactions between the particles are ignored.

Substitution of Eq。(4) into Eqs. (3a) or (3b) gives rise to four terms; in a liquid, the term proportional to $\left\langle F_{1} F_{j}\right\rangle$ ought to be expected to dominate. For the shear viscosity, this term is

$$
\begin{aligned}
(I)= & \frac{-K_{\mathrm{B}} T}{(2 \pi)^{6} V}\left\langle\int_{0}^{\infty} d t \sum_{i, j=1} r_{i x}(0) r_{j x}(t) \sum_{k_{q} q} a_{\mathrm{k}}(0) a_{\mathrm{q}}(t)\right. \\
& \left.\times k_{y} q_{y} h^{(2)}(\mathrm{k}) h^{(2)}(\mathbf{q}) \exp \left[i\left(\mathrm{k} \cdot \mathbf{r}_{i}(0)+q \circ \mathbf{r}_{j}(t)\right)\right]\right\rangle .
\end{aligned}
$$

Integration by parts and the substitution $\Delta \mathrm{r}_{i j}(t)=\mathrm{r}_{j}(t)$ $-r_{1}(0)$ yields

$$
\begin{aligned}
(I)= & \frac{K_{B} T}{(2 \pi)^{6} V}<\int d t \sum_{\substack{i j \\
\mathbf{k q}}} \exp \left[i(\mathbf{k}+\mathbf{q}) \cdot \mathbf{r}_{f}(0)+i \mathrm{q} \cdot \Delta \mathbf{r}_{i j}(t)\right] k_{y} q_{y} \\
& \times \frac{\partial}{\partial k_{x}} \frac{\partial}{\partial q_{x}}\left[a_{\mathbf{k}}(0) a_{\mathbf{q}}(t) h^{(2)}(\mathbf{k}) h^{(2)}(\mathbf{q})\right]
\end{aligned}
$$

The difficulty with further simplification of Eq. (8) is that $\Delta \mathbf{r}_{i j}(t)$ depends on both $r_{i}(0)$ and on the $a_{q}(t)$. This is readily seen for the self $(i=j)$ term. Ignoring inertial effects,

$$
\Delta \mathbf{r}_{i i}(t)=\int_{0}^{t} d \tau\left(\mathrm{v}_{B \boldsymbol{r}}(\tau)+\mathrm{F}_{i}(\tau) / f\right),
$$


$\nabla_{B r}(\tau)$ being the Brownian (solvent-induced) component of particle $i$ 's velocity and $f$ being its drag coefficient. $F_{t}(\tau)$ is given by $\mathrm{Eq}$. (5). On expanding the exponential in $\mathrm{q} \cdot \Delta r_{11}(t)$ of Eq. (8), the average over the $a_{k}(t)$ is

$$
\begin{aligned}
&\langle\{1+i \mathrm{q} \cdot\left(\int d \tau \left(\mathrm{v}_{B r}(\tau)+\frac{i K_{\mathrm{B}} T}{(2 \pi)^{3}} \sum_{p} \mathrm{p} h^{(2)}(\mathrm{p}) e^{\left(\mathrm{p} \cdot \mathrm{r}_{i}(\tau)\right.}\right.\right. \\
&\left.\left.\left.\quad \times a_{\mathrm{p}}(\tau)\right)+\cdots\right\} \frac{\partial}{\partial k_{x}} \frac{\partial}{\partial q_{x}}\left[a_{\mathrm{k}}(0) a_{\mathrm{q}}(t) h^{(2)}(k) h^{(2)}(q)\right]\right\rangle
\end{aligned}
$$

the second term of which depends on $r_{l}(0)$ through the exponential. The terms in $\left\langle\mathrm{v}_{B r} a_{\mathbf{r}} a_{\mathrm{q}}\right\rangle$ vanish because the random force $F_{s i}(t)$ due to the solvent is not correlated with the concentration fluctuations $a_{\mathbf{r}}(t)$. The random phase approximation

$$
\left\langle a_{\mathbf{k}}(0) a_{\mathrm{p}}(\tau) a_{\mathrm{q}}(t)\right\rangle=0
$$

eliminates all other terms in Eq. (10) except for the very first. In this approximation, the average over initial particle positions $r_{i}(0)$ yields $(2 \pi)^{3} \delta(k+q)$, so that the self term is

$$
\begin{aligned}
I_{s} & =\frac{-K_{B} T}{(2 \pi)^{3}} \frac{N}{V} \int d t\left\langle e^{i \mathbf{k} \cdot \Delta \mathrm{r}(t)}\right\rangle \\
& \times k_{y}^{z}\left\langle\left[\frac{\partial}{\partial k_{\mathrm{x}}} \frac{\partial}{\partial q_{\mathrm{x}}} a_{\mathbf{k}}(0) a_{\mathrm{e}}(t) h^{(2)}(\mathbf{k}) h^{(2)}(\mathbf{q})\right]\right\rangle_{\mathrm{a-k}},
\end{aligned}
$$

where the derivatives must be taken before setting $q$ $=-k$. For hard spheres of radius $R_{0}$, in the low-concentration limit

$$
h^{(2)}(\mathrm{k})=32 \pi R_{0}^{3} \frac{\sin \left(2 k R_{0}\right)-\left(2 k R_{0}\right) \cos \left(2 k R_{0}\right)}{\left(2 k R_{0}\right)^{3}}
$$

In the same limit, if $\mathrm{k}=-\mathrm{q}$,

$$
\begin{aligned}
& \left\langle a_{\mathrm{k}}(0) a_{\mathrm{q}}(t)\right\rangle=\frac{N}{V} e^{-D m^{k^{2} t}}, \\
& \left\langle e^{-i \mathrm{k} \cdot \Delta r(t)}\right\rangle=e^{-D} m^{k^{2} t}
\end{aligned}
$$

where $D_{m}=K_{B} T / 6 \pi \eta R_{0}$ is the diffusion coefficient of the particles to lowest (zeroth) order in the concentration. Corrections to $D_{m}$ due to concentration effects contribute to $I_{s}$ terms which are higher order in $\varnothing$ than those we wish to consider here.

On performing a series of elementary (albeit tedious) integrals, one obtains

$$
I_{s}=\frac{336}{25} \eta \varnothing^{2}
$$

All contributions to $\eta$ from direct interactions, other than those included in $I_{s}$, are now argued to be of order $\varnothing^{3}$ or greater. These terms, which involve the littlestudied triple dynamic structure factor

$$
S^{(3)}\left(\mathrm{k}, \mathrm{q}, t, t^{\prime}\right)=\left\langle a_{\mathbf{k}}(0) a_{\mathrm{q}}(t) a_{\mathbf{k}-\mathbf{q}}\left(t^{\prime}\right)\right\rangle,
$$

will not be considered further. Specifically, the exponential in the distinct part of Eq. (10) may be rewritten

$$
\left[\exp \left\{i(\mathbf{k}+\mathrm{q}) \cdot \mathbf{r}_{i}(0)+i \mathrm{q} \cdot\left[\mathrm{r}_{j}(0)-\mathrm{r}_{j}(0)\right]+i \mathrm{q} \cdot \Delta \mathrm{r}_{j j}(t)\right\} .\right.
$$

The factor $\exp \left[i q \cdot \Delta r_{i j}(0)\right]$ is, in the first approximation, the spatial fourier transform of the distinct part of the radial distribution function, which vanishes for noninteracting particles, and is thus of order at least $N / V$. The sum over $i$ and the factor of $a_{\mathrm{k}}(0) a_{\mathrm{q}}(t)$ each give an additional factor of $N / V$, so that the distinct part of Eq. (8) is like $(N / V)^{3}$.

Eqs. (3a), (3b) also contain terms

$$
\begin{aligned}
& \text { (II) }=\frac{1}{V}\left\langle\sum_{i, f} \mathrm{p}_{i}(t) \mathrm{p}(t) \mathrm{r}_{f}\left(t^{\prime}\right) \mathrm{F}_{f}\left(t^{\prime}\right)\right\rangle m_{i}^{-1} \\
& (\mathrm{III})=\frac{1}{V}\left\langle\sum_{i f} \mathrm{p}_{i}(t) \mathrm{p}_{i}(t) \mathrm{p}_{f}\left(t^{\prime}\right) \mathrm{p}_{f}\left(t^{\prime}\right)\right\rangle m_{i}^{-1} m_{f}^{-1}
\end{aligned}
$$

By comparison with Eq. (9), $\mathrm{p}_{f}(t)$ contains a factor proportional to $m_{i} F_{i}(t) / \zeta$. (The contribution to $p_{i}(t)$ from $v_{B r}(t)$ has the very short correlation time $\left(m_{t} / \zeta\right)$ and may be neglected.) From Eq. (5), each factor of $\mathrm{F}_{f}(t)$ yields a factor $a_{\mathbf{2}}(t) . \mathrm{Eq} .(16 \mathrm{a})$ is thus proportional to $\left\langle a_{\mathbf{k}}(t) a_{\mathbf{q}}(t) a_{\mathrm{p}}\left(t^{\prime}\right)\right\rangle$, which has been assumed to vanish. In Eq. (16b), the sum on $i$ yields a first factor of $N / V$. The average over the forces is proportional to $\left\langle a_{k}(t) a_{k}(t) a_{q}\left(t^{\prime}\right) a_{q}\left(t^{\prime}\right)\right\rangle$. Since $a_{k}(t)$ is a Gaussian random variable, this term includes both factors eliminated by the random phase approximation and factors $\left\langle\left|a_{\mathbf{k}}(t)\right|^{2}\right\rangle\left\langle\left|a_{\mathrm{a}}(t)\right|^{2}\right\rangle$, which are like $(N / V)^{2}$. The leading terms of Eq. (16b) are therefore also of order $(N / V)^{3}$. $\mathrm{Eq}$. (15) is therefore a complete expression to order $\varnothing^{2}$ for the contribution of the hard-sphere interaction to $\eta$.

An essentially identical series of arguments allows evaluation of Eq. ( $3 \mathrm{~b}$ ) for the bulk viscosity, leading to the result

$$
\eta_{v}=\eta_{r_{0}}+\frac{108}{25} \eta_{0} \phi^{2}
$$

where $\eta_{v 0}$ is the bulk viscosity of the pure fluid and $\eta_{0}$ is the viscosity factor from the diffusion coefficient.

It is instructive to compare the results obtained here [Eqs. (15), (17)] with results from theoretical hydrodynamic calculations and from experiments on sphere suspensions. The concentration dependence of the viscosity may be expanded

$$
\eta=\eta_{0}\left(1+a_{1} \varnothing+a_{2} \varnothing^{2}+a_{3} \phi^{3}+\cdots\right)
$$

Einstein predicted $^{4} a_{1}=2,5$. For $a_{2}$, a variety of values have been calculated, including $9.15,{ }^{5} 12,6,{ }^{6}$ and $14.10^{7}$ Cheng and Schachman ${ }^{8}$ report an experimental determination of $a_{1}$ and $a_{2}$ for a suspension of polystyrene latex finding good agreement with their data for $a_{1}=2.5$ and $a_{2}=14.1$. However, as Cheng and Schachman emphasized in their original paper, their measurements did not reach sufficiently high concentrations to determine $a_{3}$ with any accuracy, so there may be a substantial truncation error in the experimental determination of $a_{2}$ 。 For a suspension of glass spheres, Manley and

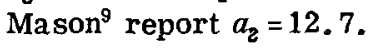

Calculated values for the hydrodynamic and contact contributions to $a_{2}$ are thus nearly the same size. Furthermore, if the hydrodynamic and direct forces are not correlated, their contributions to $\eta_{v}$ are independent and additive, in which case one predicts $a_{2} \sim 25-27$, which is somewhat larger than indicated by experiment. The discrepancy could be eliminated if the two forces were correlated, as would occur if the fluctuating hydrodynamic force on a particle were reduced by the presence of other, nearby particles. 
In many systems, the Stokes' Law drag coefficient $f$ depends linearly on the solution viscosity $\eta$, so that $f / \eta$ is a constant. If one measures $f$ indirectly, as by measuring the tracer diffusion coefficient $D_{T}=K_{B} T / f$, one continues to find

$$
D_{T} \eta=\text { constant }
$$

The calculation gives here indicates that Eq. (19) will not obtain in suspensions whose dissipative behavior includes a substantial concentration dependence due to direct interactions. From the previous calculation, ${ }^{1}$ the contact interaction makes a first order contribution to $f:$

$$
f=f_{0}\left(1+\frac{8}{3} \varnothing\right) ;
$$

in contrast, the direct interactions contribute to $\eta$ only in order $\varnothing^{2} . f / \eta$ is therefore concentration dependent.

Detecting this effect in a hard sphere system may be somewhat difficult. Hydrodynamic interactions between the Brownian particles make a contribution to $f$ as important as that of direct interactions; they also contribute to $\eta$ in order $(N / V)^{1}$. However, it was first noted by $\mathrm{MazO}^{10}$ that, in a low-salt suspension of charged particles, electrostatic interactions can increase $f$ far more effectively than the contact interaction does. An identical effect will obtain for the direct contribution to $\eta$. Since increasing the charge of a protein molecule has but a slight effect on its hydrodynamic interactions, the effects predicted above will probably be more prominent in a suspension of highly charged particles.

The results of this note appear to provide a qualitative explanation for the experimental results of Weissman and Ware, ${ }^{11}$ who studied the ionic strength dependence of the mutual diffusion coefficient $D_{m}$ and viscosity $\eta$ of a solution of bovine serum albumin of average charge $\langle z\rangle=-20$. They found that $f$ (as obtained from $D_{m}$ ) and the viscosity (as measured directly) both change with decreasing ionic strength, but that $f$ changes far more dramatically than $\eta$. In order to obtain quantitative agreement between the theory presented above and the experimental results of Weissman and Ware, it would at least be necessary to repeat the above calculations while using a form for $h^{(2)}(k)$ corresponding to a screened electrostatic interaction. This is not as easy as it might appear. Bovine serum albumin has a very large fixed electric dipole moment $(\sim 400 \mathrm{D}),{ }^{12}$ which tends to dominate any electrostatic interaction calculation, greatly complicating the averaging procedure.

\section{ACKNOWLEDGMENTS}

This calculation was motivated by discussions with B. R. Ware and M. B. Weissman in which the author's attention was drawn to their experimental results.

${ }^{1}$ G. D. J. Phillies, J. Chem. Phys. 67, 4690 (1977).

${ }^{2}$ For a detailed discussion, cf., e.g. , R. Balescu, Equilibrium and Non-Equilibrium Statistical Mechanics (Wiley, New York, 1975).

${ }^{3}$ G. D. J. Phillies, J. Chem. Phys. 60, 976 (1974); B. J. Ackerson, J. Chem. Phys. 64, 242 (1976).

${ }^{4}$ A. Einstein, Ann. Phys. 19, 289, 371 (1906); 34, 591 (1911).

${ }^{5}$ (a) V. Vand, J. Phys. Colloid Chem. 52, 277 (1948), including the corrections of (b) H. L. Goldsmith and S. G. Mason in F. R. Eirich (Ed.) Rheology, Vol. 4 (Academic, New York, 1967), p. 209.

${ }^{6}$ N. Saito, J. Phys. Soc. Jpn. 5, 4 (1950); R. Simha, J. Appl. Phys. 23, 1020 (1952).

${ }^{7}$ Cited by 5 (b) as O. Gold, Diss. Vienna (1937).

${ }^{8}$ P. Y. Cheng and H. K. Schachman, J. Polymer Sci. 16, 19 (1965).

${ }^{9}$ R. St. J. Manley and S. G. Mason, Can. J. Chem. 32, 763 (1954).

${ }^{10}$ R. Mazo, J. Chem. Phys. 43, 2873 (1965).

${ }^{11} \mathrm{M}$. B. Weissman and B. R. Ware, in preparation.

${ }^{12}$ P. Moser, P. G. Squire, and C. T. O'Konski, J. Phys. Chem. 70, 744 (1966). 